\title{
CORRIGENDUM
}

\section{Mitf is the key molecular switch between mouse or human melanoma initiating cells and their differentiated progeny}

Y Cheli, S Giuliano, T Botton, S Rocchi, V Hofman, P Hofman, P Bahadoran, C Bertolotto and R Ballotti

Oncogene (2011) 30, 2390; doi:10.1038/onc.2011.143

Correction to: Oncogene (2011) 30, 2307-2318; doi:10.1038/onc.2010.598; published online 31 January 2011
Since the publication of the above manuscript the authors have identified a spelling error in the author list. The corrected author list is shown above. 